\title{
Modelling the Cleaner Phase of a Wet High- Intensity Magnetic Separator by Employing the Levenberg-Marquardt Algorithm
}

\author{
M. Potgieter, A.F. van der Merwe, F.H. Conradie
}

\begin{abstract}
Chrome is a fundamental commodity in our everyday life, i.e. chromium is used in turbine engines, catalyst preparation, transportation and more. Conventional beneficiation thereof can be divided into comminution and concentration. The downfall to conventional beneficiation, however, entails the low efficiency of the concentration section for particle sizes inferior to $75 \mu \mathrm{m}$. This inefficiency builds up to a loss of approximately $25 \%$ of valuable materials as fines in the tailings. Alternative methods, such as the Wet High-Intensity Magnetic Separator (WHIMS), were consequently invented for further beneficiation of these tailings. This paper details an investigation into the capabilities of an artificial neural network (ANN) to predict the cleaner stage WHIMS output by employing the Levenberg-Marquardt (LM) algorithm. Both multi-input-multi-output (MIMO) and multi-input-single-output (MISO) networks were employed and the topology of each optimized. The combined performance of the MISOs proved more accurate than the MIMO network predicting with overall errors of $0.6 \%$ mean absolute error (MAE) and 0.07 root mean square error (RMSE). However, taking the mean absolute percentage error (MAPE) and the coefficient of multiple determination $\left(\mathrm{R}^{2}\right)$ into account, the MIMO network outperformed the combined MISOs, predicting with a MAPE of $3 \%$ with a correlation of 0.993 .
\end{abstract}

Keywords - Artificial neural networks, cleaner-phase WHIMS, Levenberg-Marquardt

\section{INTRODUCTION}

Chrome is a fundamental mineral product that is not easily replaceable in a variety of applications [1]. During conventional beneficiation thereof, approximately $25 \%$ of valuable material is lost as fines in the tailings [2]. A piece of beneficiation equipment invented with the purpose of further beneficiating such tailings is the WHIMS. On account of three interacting forces, i.e. magnetic, gravitational and inter particulate forces, slurry can either exit a WHIMS as a tailing (non-magnetic material), a middling or a mag (magnetic material) [3]. The WHIMS utilizes an induced gravitational field that captures magnetic material in matrices while washing off non-magnetic material. The magnetic material is hereafter washed off at the point where the magnetic field does not have an influence on the particle anymore.

Manuscript received 23 October, 2019. This work was supported in part by J. Cronje with Minprotech Rustenburg (johano@minprotech.co.za)
The measurable cleaner stage input variables include wash water flow rate, feed flow rate, slurry density, magnetic intensity, particle size, feed chrome percentage and feed chrome to iron ratio. The efficiency of the WHIMS will be defined by (i) product grade, (ii) recovery and (iii) yield.

An ANN is used to predict these outputs, due to its tailored capability to recognize patterns that other linear fitting curves will not be able to model accurately [4]. Specifically to the extraction of chromite ore, past experiments done by Reichel [5] presented results that showed that ANNs are preferred above mechanistic and statistical methods to predict the outputs based on prediction accuracy. ANNs do not predict outcomes by means of physical coding, but rather by learning characteristics of the data as a result of a pattern recognition process called training [6].

The typically used ANN is the fully connected, feed-forward layered network undergoing supervised training [7]. This type of architecture is utilized in this study. Conventional and robust algorithms include the backpropagation, Levenberg-Marquardt and scaled conjugate algorithms, with the backpropagation method by far the most commonly used algorithm [8]. The Levenberg-Marquardt algorithm was chosen due to its ability to converge quickly and in a stable manner as well as the suitability in the use of a small data set [9].

To validate the network, different performance measurements can be used, however, for networks with relatively smooth data and for the purpose of pattern recognition, it is advised to use RMSE and MAE performance measurements [10].

Each network's topology plays a cardinal role in the behavior of the network itself [6]. Numerous factors need to be taken into account when optimizing the topology, i.e. the number of hidden layers and the number of hidden neurons per hidden layer [11]. General rules of thumb exist that estimate the number of hidden neurons [12], however, additional methods to determine the optimum number of hidden neurons include Hopfield's neural network and Akaike's information criterion [11]. Plant data can also be used to validate the network's performance. Furthermore, when comparing MIMO and MISO networks, MISO networks generally outperform MIMO networks, as confirmed by Chang et al. [13].

M. Potgieter was with North West University, Potchefstroom (mirgie11@gmail.com). A.F. van der Merwe is with North West University, Potchefstroom (frikkie.vandermerwe@nwu.ac.za) 


\section{EXPERIMENTAL METHOD}

TABLE I: A LIST OF MATERIALS AND EQUIPMENT USED.

\begin{tabular}{ll}
\hline Materials & Equipment \\
\hline Raw chromite ore & Containers \\
Water & Drying oven \\
Filter paper & Oven pans \\
WHIMS rougher product & Filter press \\
& Laboratory scale \\
& Industrial scale \\
& Two-way splitter \\
& Ten-way splitter \\
& WHIMS pilot plant \\
& Sieves and shaker \\
& Collection drums \\
\hline
\end{tabular}

Materials and equipment necessary to complete these experiments are listed in Table I. A pilot plant WHIMS with model number RW9301 102 manufactured in Australia was used to execute the experiments. In order to construct a supervised learning ANN, both input and output values need to be obtained. To quantify some of the inputs, some preparation prior to the operation of the WHIMS was required. Similar experimental steps are carried out on the product to obtain output values.

\section{A. Sample Preparation}

Raw material was received in the form of a slurry from two plants situated around Rustenburg. The first step was to leave the sample to settle in order to decant as much water as possible. A period of two hours dedicated to settling has proven to be sufficient for solid-liquid separation to take place.

The following step entailed drying of the solid material. The solids were dried in an oven at a temperature of $130{ }^{\circ} \mathrm{C}$ for a period of 24 hours. For the WHIMS operation, a minimum amount of $10 \mathrm{~kg}$ of solid material is required to obtain credible results. Consequently, the total weight of the dried material was split into batches with a mass slightly higher than $10 \mathrm{~kg}$ by using the two-way splitter multiple times.

Subsequent steps entailed determination of the particle size distribution (PSD) and $\mathrm{x}$-ray fluorescence (XRF) analysis of the feed to obtain the feed particle size, chrome to iron ratio and chrome percentage. Since the bulk is now split into multiple batches with each batch representative of the bulk, material with a mass within the range of 2-3 $\mathrm{kg}$ was taken from one of the batches to the ten-way splitter to deliver a sample with a mass of 200-300 g. Only one of the ten parts was necessary to conduct the remainder of the sample preparation steps and the rest was added back into the batch it was taken from.

The 200-300 g sample needed to undergo separation based on particle sizes. Multiple sieves were used in the arrangement detailed in Table II. Water was used at a low flow rate for each individual sieve to aid the solid separation.

Due to fact that the previous step was conducted on a wet basis, the material needed to be dried before the material on each sieve could be weighed to produce the PSD. The material is again dried at $130{ }^{\circ} \mathrm{C}$ in the drying oven, however, 3 hours should be ample. Once the material was dry, the mass on each
TABle II: A List OF Sieves Used Together WiTH THE SiEVE Sizes.

\begin{tabular}{ll}
\hline Sieve number & Sieve size $(\mu \mathrm{m})$ \\
\hline 1 & 850 \\
2 & 600 \\
3 & 500 \\
4 & 300 \\
5 & 212 \\
6 & 150 \\
7 & 106 \\
8 & 75 \\
9 & 53 \\
10 & 38 \\
\hline
\end{tabular}

sieve was obtained, noted and separately stored to undergo XRF analysis. All the information required to construct a PSD and calculate the $\mathrm{d}_{50}$ (diameter at which $50 \%$ of the material will pass through) was now known. The $\mathrm{d}_{50}$ was used to represent the particle size of the bulk sample. The material weighed and stored was subsequently sent for XRF analysis.

\section{B. Operating the WHIMS}

A baseline for the operating conditions of the WHIMS is provided in Table III. Step changes in each variable are implemented in order to investigate the effect of each variable on the WHIMS' operation.

TABLE III: WHIMS OPERATING CONDITIONS BASELINE.

\begin{tabular}{lll}
\hline Variable & Baseline & Unit \\
\hline $\begin{array}{l}\text { Wash water flow rate (single stage) } \\
\text { Wash water flow rate (double }\end{array}$ & 10 & $l /$ min \\
$\begin{array}{l}\text { stage) } \\
\text { Feed water flow rate }\end{array}$ & $l /$ min \\
$\begin{array}{l}\text { Magnetic flux } \\
\text { Feed density }\end{array}$ & 7.3 & $l /$ min \\
& 13.5 & A \\
& 30 & $\begin{array}{l}\% \text { solids } \\
\text { (wt \%) }\end{array}$ \\
\hline
\end{tabular}

Before the WHIMS operation could commence, a few things needed to be verified. A $2200 l$ tank was used to add sufficient water necessary during the WHIMS test. The level thereof needs to be verified. In addition, the amount of water needed to produce a slurry containing the mass of the test batch needed to be calculated to deliver the desired density for the run.

The rotor was started and the wash water flow rate was adjusted to the desired value prior to the run.

Next, the magnetic flux was set to the desired value and the product and tailings outlet pipes were inserted into separate bins. Hereafter, the slurries were made up to the desired feed density and added to the circulation section of the WHIMS. To ensure that the slurry was thoroughly mixed, the slurry was circulated for approximately $5 \mathrm{~min}$.

After these steps have been completed, the WHIMS test can commence. The magnet was switched on and the wash water feed valve opened. The desired number of feed pipes were transferred into the hopper feeding the WHIMS. Once the circulation section containing the slurry was almost empty, water was added to flush the system.

The product analysis is similar to steps carried out for sample preparation. The chrome percentage in the product needs to be 
determined to calculate the grade, recovery $(\operatorname{Rec})$ and yield with (1) [5], (2) [14] and (3) [14] with $m_{\text {feed }}$ and $m_{\text {product }}$ referring to the mass of the feed $(\mathrm{kg})$ and mass of product $(\mathrm{kg})$ respectively. Furthermore, $\mathrm{Cr} \%_{\text {product }}$ and $\mathrm{Cr} \%_{\text {feed }}$ refer to the chrome percentage within the product and the feed respectively.

$$
\begin{gathered}
\text { Grade }=\frac{m_{\text {product }}\left(\text { Cr } \%_{\text {product }}\right)}{m_{\text {feed }}} \times 100 \\
\text { Rec }=\frac{m_{\text {product }}\left(\text { Cr } \%_{\text {product }}\right)}{m_{\text {feed }}\left(\text { Cr } \%_{\text {feed }}\right)} \times 100 \\
\text { Yield }=\frac{m_{\text {product }}}{m_{\text {feed }}} \times 100
\end{gathered}
$$

\section{Repeatability}

The repeatability of the experiments carried out on the WHIMS machinery can be presented by calculating the standard deviation from the mean obtained by repeated experiments. A run with $13.18 \mathrm{~kg}$ of solids and $60 \mathrm{~kg}$ of water making up a feed that is fed at $58 \mathrm{l} / \mathrm{min}$ with magnetic flux of $11 \mathrm{kG}$ and $15 \mathrm{l} / \mathrm{min}$ wash water flow rate were carried out seven times. The mass of the product produced each time were measured and the standard deviation from the mean was calculated with the use of (4)-(7) [15]. Here, $\mu$ refers to the mean value of the data, $N_{\text {duplicates }}$ the number of duplicates, $S E_{i}$ the squared error of the $i^{\text {th }}$ run, $\sigma$ the standard deviation and $\sigma_{\text {mean }}$ the standard deviation from the mean obtained.

$$
\begin{gathered}
\mu=\frac{\sum m_{\text {product }}}{N_{\text {duplicates }}} \\
S E_{i}=\left(m_{\text {product }_{i}}-\mu\right)^{2} \\
\sigma=\sqrt{\frac{\sum S E_{i}}{N_{\text {duplicates }}-1}} \\
\sigma_{\text {mean }}=\frac{\sigma}{\sqrt{N_{\text {duplicates }}}}
\end{gathered}
$$

\section{Outliers}

To remove any possible outliers within input and output data, a simple calculation yielding the upper and lower bounds was done with the use of (8)-(10) [53]. $Q_{3}$ and $Q_{1}$ refers to the upper (75\%) quartile and lower (25\%) quartile of the data respectively. Furthermore, $I Q R$ refers to the inter quartile range. Any value obtained from the experiments higher than the upper bound or lower than the lower bound was regarded as an outlier and discarded.

$$
\begin{gathered}
I Q R=Q_{3}-Q_{1} \\
\text { Lower bound }=Q_{1}-1.5 \times I Q R \\
\text { Upper bound }==Q_{3}+1.5 \times I Q R
\end{gathered}
$$

\section{Model DeVelopment AND Network Optimization}

\section{A. Model Development}

The first objective is to construct a code that can create an ANN with specific characteristics. The input and output data used in this code is obtained from experiments.
Multiple programs can be utilized to construct an ANN [16]. In this case, MATLAB R2017b was used. Each ANN consists of a fully connected feedforward layered network topology with one hidden layer, using the Levenberg-Marquardt learning algorithm. All of the networks required desired output values and were therefore considered to be supervised learning networks [11]. After the network has been trained with the training data set, the network's topology needed to be optimized as the effectiveness of the ANN lies within its topology [6].

\section{B. Network Optimization}

When constructing an ANN, there are five factors that need to be considered and/or determined. These factors include: (i) selecting the number of hidden layers, (ii) determining the number of hidden neurons in each hidden layer, (iii) find an optimal solution with the aim of avoiding local minima, (iv) take convergence time constraints into account and (v) validation of network on account of overtraining [11].

\section{Selecting Number of Hidden Layers}

The choice of the number of hidden layers can be made by investigating the characteristics of the data. Panchal et al. [11] explained that one hidden layer is enough for conventional problems and that the additional second layer rarely improves the network's performance. A second hidden layer should however be added when dealing with data containing discontinuities [11].

\section{Determining Number of Hidden Neurons}

As an initial estimation of the number of hidden neurons $\left(N_{h}\right)$, rules of thumb based on number of inputs $\left(n_{i n}\right)$ and outputs $\left(n_{\text {out }}\right)$ as given in (11)-(14) can be used [11], [12]. However, this is a mere starting point. The optimum number of hidden neurons can be determined with different selection approaches, including: (i) a simple method, (ii) Hopfield neural network or (iii) Akaike's Information Criterion (AIC) [11].

$$
\begin{gathered}
N_{h}=n_{\text {in }}+n_{\text {out }}-0.5 \\
n_{\text {out }}<N_{h}<n_{\text {in }} \\
N_{h}=\frac{2}{3} n_{\text {in }}+n_{\text {out }} \\
N_{h}<2 n_{\text {in }}
\end{gathered}
$$

(i) Simple method: The configuration for a backpropagation method can be denoted as $l-m-n$, with $l$ referring to the number of input neurons, $m$ the number of hidden neurons and $n$ the number of output neurons. Here $m$ is chosen to be smaller than the number of input neurons but larger than the number of output neurons [11].

(ii) Derived from the Hopfield neural network: The Hopfield network is one of the simplest neural networks consisting of an input, output and single hidden layer with a fully connected neuron structure. It dictates that the number of hidden neurons can be set equal to the number of input neurons [11].

(iii) Akaike's Information Criterion (AIC): The AIC is a criterion based on entropy concepts that provides a measurement of the goodness of the statistical model. It describes the trade-off between bias and variance. The ANNs with different number of hidden neurons, can be ranked according to their AIC, with the lowest AIC indicating the best 
configuration. The formula to calculate the AIC is given in (15) [11].

$$
\text { AIC }=-2 \times \ln (\text { likelihood })+2 \times k
$$

Where likelihood refers to maximum log likelihood of the model and $k$ refers to the number of free parameters in the model. Additionally, AIC can also be calculated with (16) [17], where $n$ is the number of data points.

$$
A I C=n \times \ln \left(\frac{S S E}{n}\right)+2 \times k
$$

After the AIC has been calculated, the credibility of how good each network approximates actual data should be quantified. The first step to quantify this is to calculate how far each network's AIC is from the minimum obtained AIC as seen in (17) [11].

$$
\Delta i=A I C_{i}-\min \left(A I C_{i}\right)
$$

Next, an estimation of the likelihood of the model can be obtained with (18) [11].

$$
L(\text { model }) \propto \exp \left(-0.5 \Delta_{i}\right)
$$

For optimal interpretation of the likelihood estimation, the values obtained in (18) should be normalized with the use of (19). This is called the Akaike's weight $\left(A W_{i}\right)$ of each model [11].

$$
A W_{i}=\frac{\exp \left(-0.5 \Delta_{i}\right)}{\sum_{i=1}^{n} \exp \left(-0.5 \Delta_{i}\right)}
$$

The candidate models can now be filtered out by the general rule of thumb (see (20)) stating that any model with an Akaike weight greater than $10 \%$ of the largest weight is plausible to be a candidate model [11].

$$
0.1 \times \max \left(A W_{i}\right)<\text { Cand models }
$$

The AIC will be considered as the determining factor when deciding on the optimum number of hidden neurons.

3. Finding an Optimal Solution with the Aim of Avoiding Local Minima

Conventional ANN architectures contain a feed forward network using a backpropagation algorithm, which is also the case in this study. However, the downfall of the backpropagation algorithm is that it may sometimes move to a local minimum and not the overall (global) minimum [18].

To overcome this occurrence, numerous studies have been conducted to propose solutions [18]. The widest used method is merely to iteratively train the network with initial random weights [19]. This, however, can be time consuming. In a paper authored by Atajulreka and Sutivong [18], the use of Resilient Backpropagation method, which is more time efficient, yet very effective, is detailed.

To simplify things, the rules of thumb are used to obtain an upper and a lower boundary for the number of hidden neurons applicable to this study. AIC results are also to be obtained within these limits, otherwise the limits are extended accordingly. Hereafter the network is trained 10 times for each number of hidden neurons until a greater certainty exists that the backpropagation algorithm focused on the global minima and not the local minima.

\section{Convergence Time Constraints}

In certain cases, the time the network takes to converge can play a cardinal role in the choice of number of hidden neurons
[39]. This network, however, comprises of very little data and convergence takes place in under a second for a network containing 100 hidden neurons, which therefore eliminates the need to account for a time constraint.

5. Validation of Network on Account of Overtraining

When validating an ANN, the correct error measurement should be chosen. A clear-cut theoretical approach to validate a neural network unfortunately does not exist, however, four conventionally used performance measures include the MAE, MAPE, RMSE and mean square error (MSE). Specifically, for networks containing smooth output vectors and for the purpose of pattern classification, the MAE and RMSE are usually used to validate the network's performance [10]. The MAPE, together with the coefficient of multiple determination $\left(\mathrm{R}^{2}\right)$, is used to assess the performance of the networks.

When training neural networks, it is a challenge to stop the training just before overfitting occurs and the network starts to lose its generalization abilities, yet leaving enough room for the training to learn the data set properly [20]. One approach to avoid overtraining is to investigate the number of epochs. It is treated as a hyperparameter while training the network multiple times, where-after an epoch is chosen that produces the best performance [10].

Another approach is called early stopping, where the generalization error of the network is continuously being calculated and the training is stopped when this error starts to increase [10]. Fortunately, this method is already accounted for when using MATLAB ${ }^{\mathrm{TM}}$ to construct supervised feedforward neural networks [21].

\section{RESULTS AND DisCUSSIONS}

\section{A. Repeatability and Outliers}

The WHIMS operation was repeated 7 times and presented a $75 \mathrm{~g}$ standard deviation from the mean when using $73.18 \mathrm{~kg}$ feed (slurry). The WHIMS therefore showed an acceptable repeatability. Furthermore, all the data were tested for outliers and none of the data points were found to be above or below the upper and lower bounds.

\section{B. MIMO Networks}

Due to the data being of a continuous nature, 1 hidden layer was used.

The general rules of thumb, together with the simple method and Hopfield's neural network results, are given in Table IV. An overall range of 3 to 14 hidden neurons was obtained.

The minimum AIC resulted in a value of -399.2 using 9 hidden neurons. On account of the rule of thumb given in (20), this network was the only viable candidate.

Furthermore, performance measurements MAPE and $\mathrm{R}^{2}$ are investigated within the range of 3 to 14 neurons. With 9 hidden neurons, the AIC will be at its minimum, which also delivers a $3 \%$ MAPE and 0.993 correlation. However, when only taking the MAPE together with the $\mathrm{R}^{2}$ values into consideration, 11 hidden neurons displays the best performance. As mentioned, the AIC is chosen to be the deciding factor with regards to the optimum number of hidden neurons. Therefore, the MIMO 
TABLE IV: RESUlTS FOR THE NUMBER OF HIDDEN NEURONS ESTIMATED.

\begin{tabular}{llll}
\hline $\begin{array}{l}\text { Rule of } \\
\text { thumb }\end{array}$ & $\begin{array}{l}\text { Minimum } \\
\text { number of } \\
\text { hidden } \\
\text { neurons }\end{array}$ & $\begin{array}{l}\text { Fixed number } \\
\text { of hidden } \\
\text { neurons }\end{array}$ & $\begin{array}{l}\text { Maximum } \\
\text { number of } \\
\text { hidden } \\
\text { neurons }\end{array}$ \\
\hline 1 & 3 & 10 & 7 \\
2 & & 8 & 14 \\
3 & 3 & & 7 \\
4 & & 7 & \\
$\begin{array}{l}\text { Simple } \\
\text { method }\end{array}$ & & & \\
Hopfield & & & \\
\hline
\end{tabular}

network with 7 inputs, 9 hidden neurons and 3 output neurons is regarded as the optimum network structure according to AIC.

Hereafter, local minima is tested. After iteratively training the network each time with randomized weights, the smallest AIC obtained remains -399.2 with the use of 9 hidden neurons. It can therefore be stated with greater certainty that the minimum originally found represents a global minimum.

Taking local minima into account, the optimum MIMO network, therefore, contains 1 input layer with 7 neurons, 1 hidden layer with 9 hidden neurons and 1 output layer with 3 neurons. The performance of this network can be seen in Fig. 1.

This network presents an overall performance of $3 \%$ MAPE with an $\mathrm{R}^{2}$ of 0.993 . The grade prediction is more accurate than the predictions for recovery and yield. This could be on account of less variance found in grade data.

\section{MISO Networks}

Three different types of MISO networks were constructed. All seven inputs were used in each MISO to investigate the effect that each has on grade, recovery and yield individually.

Similar to MIMO networks, the data remains to be of a continuous nature as it is the same data used. Therefore, 1 hidden layer was used for all the MISO networks.

The general rules of thumb, together with the use of the simple method and Hopfield's neural network, resulted in an overall range of 1 to 14 hidden neurons. The minimum AIC value for the yield, recovery and grade output MISOs resulted in values of -235 with 14 hidden neurons, -549 with 5 hidden

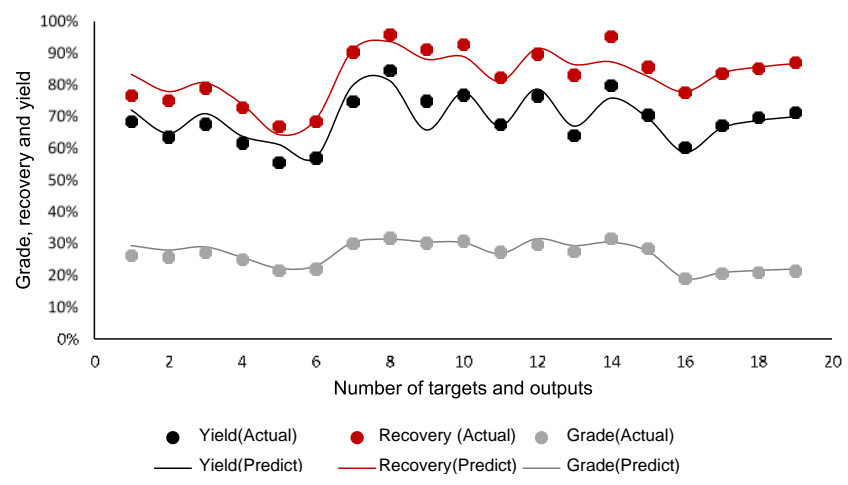

Fig. 1. Fit of optimum MIMO neural network with actual experimental output data.
TABle V: Optimum Number of Hidden NeURONS BASED ON PERFORMANCE MEASUREMENTS FOR ALL MISO NETWORKS

\begin{tabular}{llll}
$\begin{array}{l}\text { Performance } \\
\text { measurement }\end{array}$ & $\begin{array}{l}\text { MAPE } \\
(\%)\end{array}$ & $\mathrm{R}^{2}$ & $\begin{array}{l}\text { Number of } \\
\text { hidden neurons }\end{array}$ \\
\hline Yield & & & \\
$\begin{array}{l}\text { Minimum MAPE } \\
\text { Maximum R }\end{array}$ & 2 & 0.951 & 2 \\
$\begin{array}{l}\text { Recovery } \\
\text { Minimum MAPE }\end{array}$ & 4 & 0.986 & 9 \\
$\begin{array}{l}\text { Maximum R } \\
\text { Grade }\end{array}$ & 4 & 0.983 & 4 \\
$\begin{array}{l}\text { Minimum MAPE } \\
\text { Maximum R }\end{array}$ & 1 & 0.998 & 19 \\
\hline
\end{tabular}

neurons and -216 with 21 hidden neurons respectively. These networks were the only viable candidates. The optimum number of hidden neurons based on the MAPE and $\mathrm{R}^{2}$ performance measurements are given in Table V.

Similar to the MIMO network, each MISO was trained 10 times, starting each time with random weights for neurons 1 to 21 to ensure that the values obtained are not representative of local minima. The hidden neuron range is extended to investigate up until 21 neurons on account of the optimal AIC found at 21 for the grade network. The minimum AICs obtained after iteratively training the networks are $-502.5,-520$ and -405 for the yield, recovery and grade networks respectively. Comparing these values to the values initially obtained. both the yield and the grade networks obtained smaller AICs at 6 and 8 hidden neurons. This can be an indication that the grade and yield networks initially converged to local minima. In conclusion, the optimal network structures for each MISO can now be given with greater certainty as 6,5 and 8 hidden neurons for the yield, recovery and grade neural networks respectively. Each network's performance is presented in Table VI. The performance graphs are shown in Fig. 2.

TABLE VI: PERFORMANCES OF EACH OPTIMUM MISO NETWORK.

\begin{tabular}{lll}
\hline Output & MAPE $(\%)$ & $\mathrm{R}^{2}$ \\
\hline Yield & 3 & 0.971 \\
Recovery & 5 & 0.952 \\
Grade & 3 & 0.981 \\
\hline
\end{tabular}

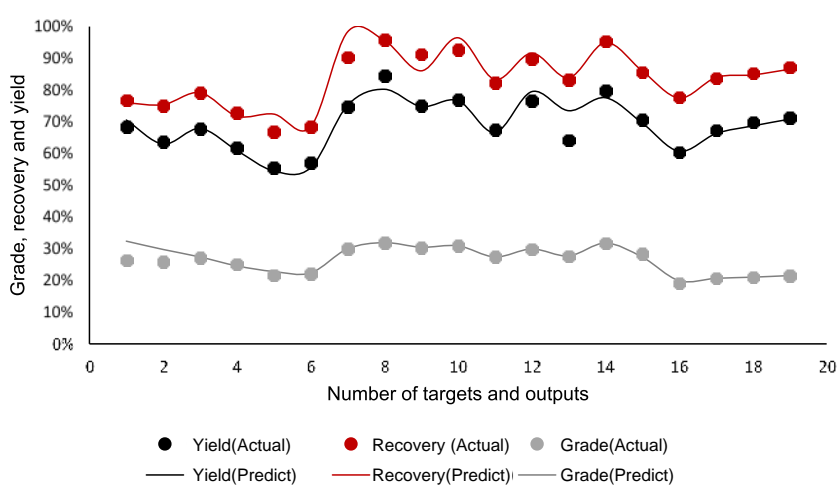

Fig. 2. Fit of optimum MISO networks to experimental data. 
TABLE VII: COMPARISON OF PERFORMANCE MEASUREMENTS MIMO NETWORKS WITH MISO NETWORKS.

\begin{tabular}{lllllllll}
\hline Output & \multicolumn{3}{l}{$\begin{array}{l}\text { MAE } \\
(\%)\end{array}$} & & RMSE & \multicolumn{1}{l}{$\begin{array}{l}\text { MAPE } \\
(\%)\end{array}$} & $\mathrm{R}^{2}$ & \\
\cline { 2 - 9 } & $\mathrm{M}$ & $\mathrm{S}$ & $\mathrm{M}$ & $\mathrm{S}$ & $\mathrm{M}$ & $\mathrm{S}$ & $\mathrm{M}$ & $\mathrm{S}$ \\
\hline Yield & 0.1 & 0.1 & 0.03 & 0.02 & & 3 & & 0.97 \\
Recovery & 0.4 & 0.3 & 0.03 & 0.05 & & 5 & & 0.95 \\
Grade & 0.5 & 0.2 & 0.01 & 0.004 & 3 & & 0.98 \\
Overall & 1.0 & 0.6 & 0.07 & 0.07 & 3 & 3.7 & 0.99 & 0.97 \\
\hline
\end{tabular}

\section{A. Comparison Between MIMO and MISO Networks}

The optimum MIMO and MISO network performances are presented in Table VII. When considering MAE and RMSE, the MISO networks primarily outperforms the MIMO, confirming findings from literature [13]. However, when considering the MAPE and $\mathrm{R}^{2}$ values, the MIMO network perform better than the combined performance of the MISO networks.

\section{VALIDATION OF NETWORK TOPOLOGY WITH PlANT DATA}

In order to inspect the validity of the chosen network and its topology, three months' plant data was used as input and output values. The combined MAPE for the MIMO and MISO networks were $10 \%$ and $4 \%$ respectively. The MIMO and MISO obtained an $\mathrm{R}^{2}$ of 0.97 and 0.79 respectively.

\section{CONCLUSIONS AND RECOMMENDATIONS}

The findings correspond with work done by Reichel [7] that showed the suitability of an ANN to model the rougher phase of a WHIMS. It is also fit to use an ANN to model the cleaner phase of the WHIMS as it models experimental data with overall errors of $0.2 \%$ MAE, 0.02 RMSE and $3.7 \%$ MAPE while displaying a correlation of 0.97 when using MISO networks. MISO neural networks are deemed optimum between MIMO and MISO networks considering performance criteria MAE and RMSE. Contrastingly, the MIMO network slightly outperformed the MISOs when taking the MAPE and $R^{2}$ into consideration. Optimum number of hidden neurons for the MISO networks are 6, 5 and 8 hidden neurons for yield, recovery and grade respectively, while the MIMO network performed optimally with 9 hidden neurons.

Some recommendations to improve on the accuracy of the model are (i) to obtain a larger dataset to allow training to take place with more data points, (ii) a more in depth study to ensure that all possible local minima are avoided and (iii) to combine the performance measurements with the AIC results to make a decision regarding the optimum number of hidden neurons

\section{REFERENCES}

J. O. Nriago and E. Nieboer, Chromium in the natural \& human environments. Wiley, 1988.

[2] N. Gence, "Beneficiation of elazig-kefdag chromite by multi-gravity separator,” J. Eng. Environ. Sci., vol. 23, pp. 473-475, 1999.

[3] J. A. Oberteuffer, "Magnetic Separation: A Review of Principles, Devices, and Applications," IEEE Trans. Magn., vol. 10, no. 2, pp. 223-238, 1974.

[4] C. Stergiou and D. Siganos, "Neural Networks.” 2019.
[5] C. R. M. Reichel, A. F. Van Der Merwe, and J. Cronje, "A Predictive Model for Wet High Intensity Magnetic Separator (WHIMS) using Artificial Neural Networks," 2018.

[6] R. Beresford and S. Agatonovic-Kustrin, "Basic concepts of artificial neural network ( ANN ) modeling and its application in pharmaceutical research," J. Pharm. Biomed. Anal., vol. 22, no. 5, pp. 717-727, 2000

[7] R. Aggarwal and Y. Song, "Artificial neural networks in power systems. Part 1: General introduction to neural computing," Power Eng. J., vol. 11, no. 3, pp. 129-134, 2005.

[8] Ö. Kişi, "Streamflow Forecasting Using Different Artificial Neural Network Algorithms," J. Hydrol. Eng., vol. 12, no. 5, pp. 532-539, 2007.

[9] H. Yu and B. M. Wilamowski, "Levenberg-Marquardt Training," Intell. Syst., pp. 12-1-12-16, 2019.

[10] J. M. Twomey and A. E. Smith, "Validation and verification," Artif. Neural Networks Civ. Eng. Fundam. Appl., no. January 1995, pp. 4464, 1997.

[11] G. Panchal, A. Ganatra, Y. P. Kosta, and D. Panchal, "Behaviour Analysis of Multilayer Perceptronswith Multiple Hidden Neurons and Hidden Layers," Int. J. Comput. Theory Eng., vol. 3, no. 2, pp. 332-337, 2011.

[12] S. Trenn, "Multilayer perceptrons: approximation order and necessary number of hidden units," IEEE Trans. Neural Networks, vol. 19, no. 5, pp. 836-844, 2008.

[13] F. J. Chang, Y. M. Chiang, and L. C. Chang, "Multi-step-ahead neural networks for flood forecasting," Hydrol. Sci. J., vol. 52, no. 1, pp. 114-130, 2007.

[14] B. A. Wills and T. Napier-Munn, Wills' Mineral Processing Technology, no. October. 2005.

[15] C. Deziel, "How do I calculate repeatability?," Sciencing, 2018. [Online]. Available: https://sciencing.com/do-calculaterepeatability-7446224.html. [Accessed: 10-Oct-2019].

[16] R. M. Yoo, H. Lee, K. CHow, and H. S. Lee, "Constructing a NonLinear Model with Neural Networks for Workload Characterization," IEEE, pp. 150-160, 2006.

[17] H. Akaike, "Fitting Autoregressive Models for Prediction," Ann. Inst. Stat. Math., vol. 21, pp. 243-247, 1969.

[18] A. Atakulreka and D. Sutivong, "Avoiding local minima in feedforward neural networks by simultaneous learning," Lect. Notes Comput. Sci. (including Subser. Lect. Notes Artif. Intell. Lect. Notes Bioinformatics), vol. 4830 LNAI, pp. 100-109, 2007.

[19] M. S. Iyer and R. R. . Rhinehart, "A Method to Determine the Required Number of Neural- Network Training Repetitions," IEEE Trans. Neural Networks, vol. 10, pp. 427-432, 1999.

[20] J. Brownlee, "A Gentle Introduction to Early Stopping to Avoid Overtraining Neural Networks," Machine Learning Mastery, 2019. [Online]. Available: https://machinelearningmastery.com/earlystopping-to-avoid-overtraining-neural-network-models/.

[21] (MathWorks), "Improve Shallow Neural Network Generalization and Avoid Overfitting," 2019. [Online]. Available: Improve Shallow Neural Network Generalization and Avoid Overfitting. [Accessed: 08-Oct-2019]. 